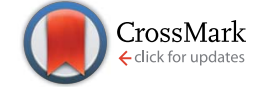

Cite this: RSC Adv., 2014, 4, 41348

Received 8th August 2014 Accepted 27th August 2014

DOI: $10.1039 / c 4 r a 08382 b$

\section{Vinylphenylglycidyl ether-based colloidal architectures: high-functionality crosslinking reagents, hybrid raspberry-type particles and smart hydrophobic surfaces $\uparrow$}

\author{
S. Mehlhase, $\star^{a}$ C. G. Schäfer, $\star^{a}$ J. Morsbach, ${ }^{b}$ L. Schmidt, ${ }^{a}$ R. Klein, ${ }^{a} H$. Frey ${ }^{\star b}$ \\ and M. Gallei*a
}

www.rsc.org/advances

An efficient synthetic strategy for the preparation of monodisperse colloidal core/shell architectures with reactive epoxy functionalities is reported, based on the bifunctional vinylphenylglycidyl ether monomer. Accessibility of the reactive immobilized epoxy moieties was quantified by UV/vis spectroscopy in an isorefractive medium. Inorganic-organic hybrid raspberry-type architectures revealed a tremendous inpact on the surface wettability of water.

The design and control of hierarchical particle-based architectures play a key role in current polymer and colloid science in order to mimic sophisticated structures known from nature. Biomimetic strategies have created enormous potential for the development of multi-dimensional materials, featuring tailor made nano-scaled architectures for optical, electrical and magnetic applications. ${ }^{1-4}$ Moreover, the control and understanding of (switchable) surface properties are crucial for the development of advanced materials with well-defined wetting properties. Such so-called smart surfaces have already been used in applications as self-cleaning surfaces, tunable optical lenses, lab-on-chip systems, microfluidic devices and for different textile applications. ${ }^{5-10}$ Considerable effort has been made to understanding the influence of designed, rough surfaces on the wetting properties, initiated by the pioneering works of Wenzel, Cassie and Baxter some time ago. ${ }^{11,12}$ In several recent studies,

${ }^{a}$ Ernst-Berl-Institut für Technische und Makromolekulare Chemie, Technische Universität Darmstadt, Alarich-Weiss-Str. 4, D-64287 Darmstadt, Germany. E-mail: m.gallei@mc.tu-darmstadt.de

${ }^{b}$ Institute of Organic Chemistry, Organic and Macromolecular Chemistry, Johannes Gutenberg-Universität, Duesbergweg 10-14, D-55099 Mainz, Germany. E-mail: hfrey@uni-mainz.de

$\uparrow$ Electronic supplementary information (ESI) available: Additional data on materials, instrumentation, general experimental procedures, DSC experiments, silica particle syntheses, detailed calculation of epoxy functionalities, TEM images, photographs of the particle dispersion after NBP treatment, UV/vis spectra, calibration plots for UV/vis measurements, additional DLS measurements, SEM images of raspberry-particle films. See DOI: 10.1039/c4ra08382b

\$ These authors contributed equally. raspberry-architectures consisting of a larger core particle surrounded by surface-attached smaller particles have especially been observed to provide high and adjustable surface roughness and high specific surface areas. This effect is also referred to as the "lotus effect", and such materials are appealing for superhydrophobic coatings and films. ${ }^{13-18}$ Generally, raspberrylike particles have been obtained via Pickering emulsion polymerization, ${ }^{19,20}$ miniemulsion polymerization, ${ }^{21-23}$ heterocoagulation $^{24,25}$ or direct hydrolysis of an inorganic precursor at the particle surface. ${ }^{26,27} \mathrm{An}$ interesting and convenient approach for formation of raspberry-type structures was recently reported by Zhao and Middelberg by taking advantage of microfluidic synthetic protocols. ${ }^{28}$

In general, modification of monodisperse particles proves feasible, if bifunctional monomers (e.g. bearing both a vinylic and an epoxy functionality) are used, maintaining at least one reactive moiety subsequent to polymerization. For this purpose, especially the bifunctional glycidyl methacrylate (GMA) has been used as a monomer in order to prepare core/shell architectures or gels with reactive epoxy groups for further functionalization. The advantage of using epoxy groups is their convenient conversion into diols, amines or aldehydes. Hence, GMA has been polymerized and used for subsequent protein immobilization, ${ }^{29,30}$ drug delivery from PGMA gels, ${ }^{31}$ for liquid chromatography, ${ }^{32,33}$ for self-healing materials, ${ }^{34,35}$ for the formation of macroporous hydrogels, ${ }^{36}$ as a polylactide toughening agent, ${ }^{37}$ as membrane adsorber ${ }^{38}$ or for photonic crystals. ${ }^{39}$ GMA has also been used in order to produce functional (hybrid) core/shell and raspberry-like architectures for different applications. ${ }^{40-47}$

Recently, the novel bifunctional monomer vinylphenylglycidyl ether (VPGE) bearing both styrenic and epoxy functionalities has been presented in a patent. ${ }^{48}$ Compared to PGMA, PVPGE features superior thermal stability and a higher chemical and hydrolytic stability. In this work we present a strategy for the one-pot preparation of VPGE-based functional core/shell particles using a seeded emulsion polymerization approach, which is advantageous compared to established 
procedures. Moreover, another focus of this work is the quantification of the reactive epoxy sites at the latex particles. The epoxy moieties in the particle shell have also been employed for subsequent crosslinking reactions. Functional, hierarchically structured raspberry-like particles have been developed and deposited on surfaces in order to adjust wettability, aiming at highly hydrophobic surfaces.

In order to prepare core/shell particles featuring a reactive functional shell based on VPGE, semi-continuous and stepwise emulsion polymerization protocols were applied, as depicted in Fig. 1 and described in the experimental section (ESI $\dagger$ ).

This procedure allows precise control over the polymer composition and the shell constitution. ${ }^{49-51}$ For this purpose, poly(butyl acrylate) (PBA) seeds were synthesized in a batch process in the first step followed by the continuous addition of styrene (S) and butyl acrylate (BA) in the second step. VPGE bearing epoxy moieties was introduced in the last step of the particle synthesis by adding a mixture of VPGE and BA, as is also described in the experimental section. In order to maintain the epoxy functionalities without significant ring-opening reaction, a mixture of sodium dihydrogen phosphate and sodium hydrogen carbonate was used as a buffer system during the seeded emulsion polymerization. The success of the core/shell particle synthesis was verified by transmission electron microscopy (TEM) measurements for the final particle dispersion (Fig. 2, left). A corresponding TEM image of the soft core particles is given in the ESI Fig. S1. $\dagger$

In addition to TEM measurements, dynamic light scattering (DLS) measurements for all particle synthesis steps were carried out (Fig. 2, right), furnishing proof for the successful generation of PVPGE-containing core/shell particles, as confirmed by the increasing average particle diameter. Moreover, corresponding particle size distributions after each reaction step revealed that monodisperse particles with a final average diameter of $348 \mathrm{~nm}$ were obtained. Values for the obtained average particle diameters for all syntheses steps are compiled in Table S2 of the ESI. $\dagger$

It is a key issue, whether the epoxide moieties of the VPGEunits are still present and reactive subsequent to emulsion polymerization. For this purpose, two strategies were followed: (i) UV/vis spectroscopy measurements in an isorefractive solvent for the particle dispersion after attachment of a dye and (ii) differential scanning calorimetry (DSC) measurements of VPGEcore/shell particles in the presence of a crosslinker, as described in the ensuing section. In general, determination of the amount

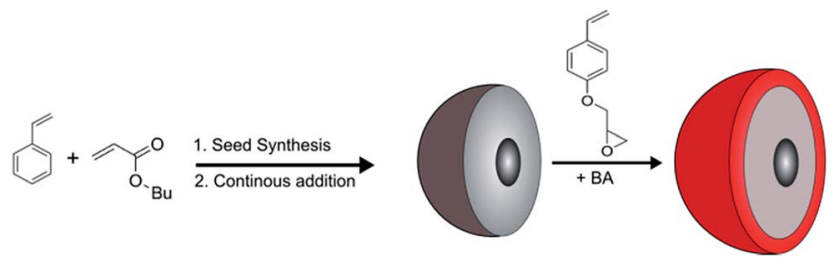

Fig. 1 Successive core/shell particle synthesis starting from poly(butyl acrylate) (PBA) seed particles followed by the continuous addition of butyl acrylate (BA) and styrene (S) and the final addition of BA and VPGE monomer.
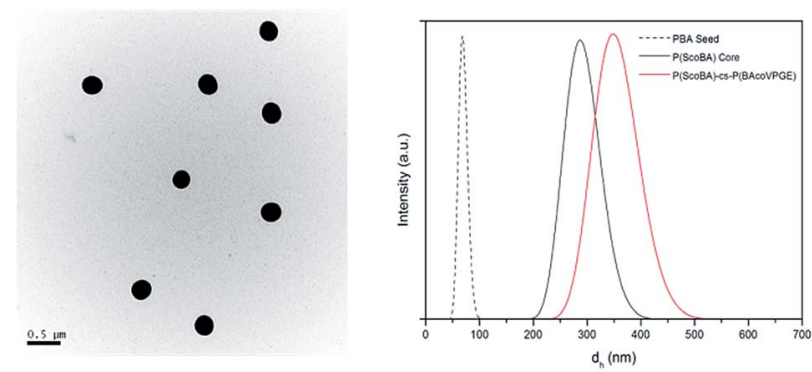

Fig. 2 Left: TEM image of $P(S-c o-B A)$ core $P(B A-c o-V P G E)$ shell particles. Scale bar corresponds to $500 \mathrm{~nm}$. Right: DLS results for the step-wise prepared $\mathrm{P}(\mathrm{BA})$ seed (dotted line), $\mathrm{P}(\mathrm{S}-\mathrm{co}-\mathrm{BA})$ core particles (black solid line) and the epoxy-functionalized PVPGE-containing core/shell particles (red line).

of functional groups at organic micro- and nanoparticles is a challenging task and typical analytical methods, such as elemental analysis, attenuated total reflectance Fourier transform infrared spectroscopy (ATR-FTIR), X-ray photoelectron spectroscopy (XPS), solid-state NMR, or calorimetric, colorimetric and fluorometric methods were used. ${ }^{52,53}$ Recently, we were able to show that transferring originally invisible ATRP initiators attached to organic particles into a RAFT chain transfer agent entity with a typical absorption band is a valuable technique in order to determine the amount of accessible ATRPinitiator by using UV/vis spectroscopy. ${ }^{\mathbf{5 2 , 5 4}}$ For this purpose it was necessary to use an isorefractive dispersion medium for the investigated nanoparticles. Notably, accessibility in this particular case strongly depends on the size of the compounds to be attached and the molecular and local environment.

In the current study we choose 4-(4-nitrobenzyl)pyridine (NBP) - also referred to as the Preussmann Reagent - for efficient ring-opening reaction of epoxy functionalities (Fig. 3).$^{55-57}$

Ring-opening reaction of NBP with surface-attached epoxy moieties at the organic particle surface or shell, respectively, results in an intensive purple coloration. Recently, Schönherr and co-workers successfully applied the Preussmann test for determining the amount of epoxy functionalities at various inorganic substrates. ${ }^{55}$ We now expand these findings to the determination of epoxy functionalities in the exterior of a polymeric particle shell by using anisole as an isorefractive solvent for the particle dispersion, relying on UV/vis spectroscopy. Exemplarily, images of the Preussmann reagent in a solution of ethanol, particle dispersion after treatment with the

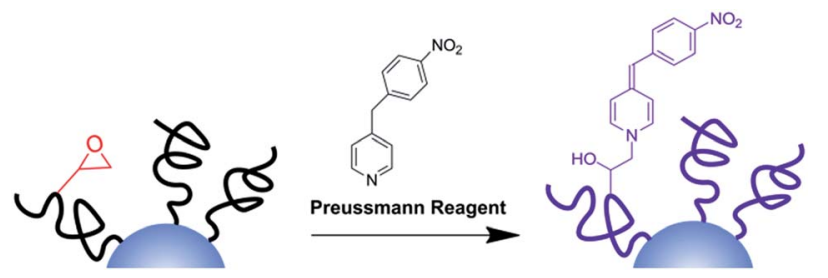

Fig. 3 Reaction of surface-immobilized epoxy moieties with 4-(4nitrobenzyl)pyridine (NBP, Preussmann reagent) for organic core/shell particle coloration. 
Preussmann reagent and the corresponding diluted particle dispersion in an isorefractive solvent are shown in Fig. S3 of the ESI. $\dagger$ By further dispersing the pristine particle dispersion in anisole, the particles became nearly invisible, since the refractive index of the organic particles and the solvent (refractive index $_{\text {anisole }}=1.516$ ) are very close. Consequently, the recorded UV/vis absorption spectra of the heterogeneous system indicated a suppression of particle scattering, enabling the quantification of epoxy functionalities (Fig. S4†). A detailed calculation of accessible epoxy functionalities after reaction with NBP is described in the ESI. $\uparrow$ The molar extinction coefficient $\varepsilon$ for NBP in anisole at $544 \mathrm{~nm}$ was determined to 1537.36 $\mathrm{cm}^{2} \mathrm{mmol}^{-1}$ from the corresponding calibration plot (Fig. S5 $\dagger$ ). After reaction with NBP, the amount of accessible epoxy functionalities, $\mathrm{c}_{\text {chromo, }}$ could be determined by UV/vis measurements. By assuming an average particle diameter of $350 \mathrm{~nm}$, a value of 5.3 epoxide moiety per $\mathrm{nm}^{2}$ could be calculated, which is in excellent agreement with expectation.

To further confirm the maintained reactivity of surfaceattached epoxides at the particles subsequent to emulsion polymerization, exothermic ring-opening reactions of PVPGEcontaining core/shell particles with 3-aminomethyl-3,5,5-trimethylcyclohexylamine as a commercially available cross-linking reagent - also referred to as isophorone diamine (IPDA) were studied using DSC measurements. Samples with different amounts of epoxy functionalities in the particle shell were treated with IPDA, as described in the experimental section (ESI $\dagger$ and Fig. S6 $\dagger$ ). In the first DSC run, an exothermic peak at approximately $70{ }^{\circ} \mathrm{C}$ due to the crosslinking reaction was obvious (Fig. S7, $\dagger$ top) demonstrating that immobilized epoxy functionalities were capable of undergoing ring-opening reaction with IPDA. Furthermore, in the second subsequent DSC experiment (Fig. S7, $\dagger$ bottom) of the same sample, a significant shift of the original glass transition temperature of $58{ }^{\circ} \mathrm{C}$ for the pristine organic particles (dotted line in Fig. S5†) to $95{ }^{\circ} \mathrm{C}$ was observed. These results additionally evidence fast and efficient ring-opening reaction of PVPGE-containing particles with IPDA. The possibility of subsequent crosslinking due to the presence of reactive epoxy-groups in the particle shell is an important tool for additional particle modification.

The PVPGE-containing core/shell particles were functionalized with smaller silica particles in order to generate raspberrylike particle architectures (Fig. 4). To this end, PVPGE-containing core particles with a high concentration of accessible surface epoxide moieties were slowly added to amino-functionalized silica particles. For this purpose, dropwise addition of a diluted core particle dispersion $(0.13 \mathrm{wt} \%$ in ethanol) to the

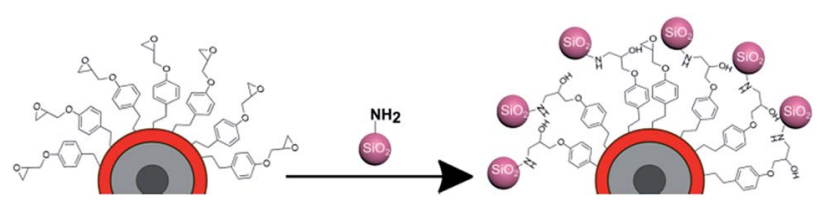

Fig. 4 Reaction of PVPGE-containing core/shell particles bearing reactive epoxy functionalities and amino-functionalized silica particles for the preparation of raspberry-like hybrid architectures. silica nanoparticle dispersion ( $2 \mathrm{wt} \%$ in ethanol) was used, resulting in a ring-opening reaction of the epoxide moieties by the surface-anchored amine groups. Average diameters of the silica nanoparticles employed were determined to $50 \pm 7 \mathrm{~nm}$ and $120 \pm 14 \mathrm{~nm}$, respectively, by using DLS (Fig. S8 $\dagger$ ) and to $48 \pm 5 \mathrm{~nm}$ and $104 \pm 5 \mathrm{~nm}$, respectively, by using TEM measurements (Fig. S9†).

Subsequent to this reaction, the excess of the smaller silica nanoparticles was removed by centrifugation, yielding rather pure hybrid raspberry-like particles consisting of an organic core and a large number of smaller silica particles. The colloidal organic-inorganic "raspberries" created have been investigated by TEM and SEM measurements (Fig. 5).

For surface modification and contact angle (CA) measurements, the raspberry-like particles (348 nm PVPGE particles with $120 \mathrm{~nm}$ silica particles) were deposited on glass wafers by coating with a doctor blade, (details see experimental section). After exposure to oxygen plasma in order to generate homogeneously distributed hydroxyl functionalities, the film was treated with dichlorodimethylsilane for hydrophobization. Both

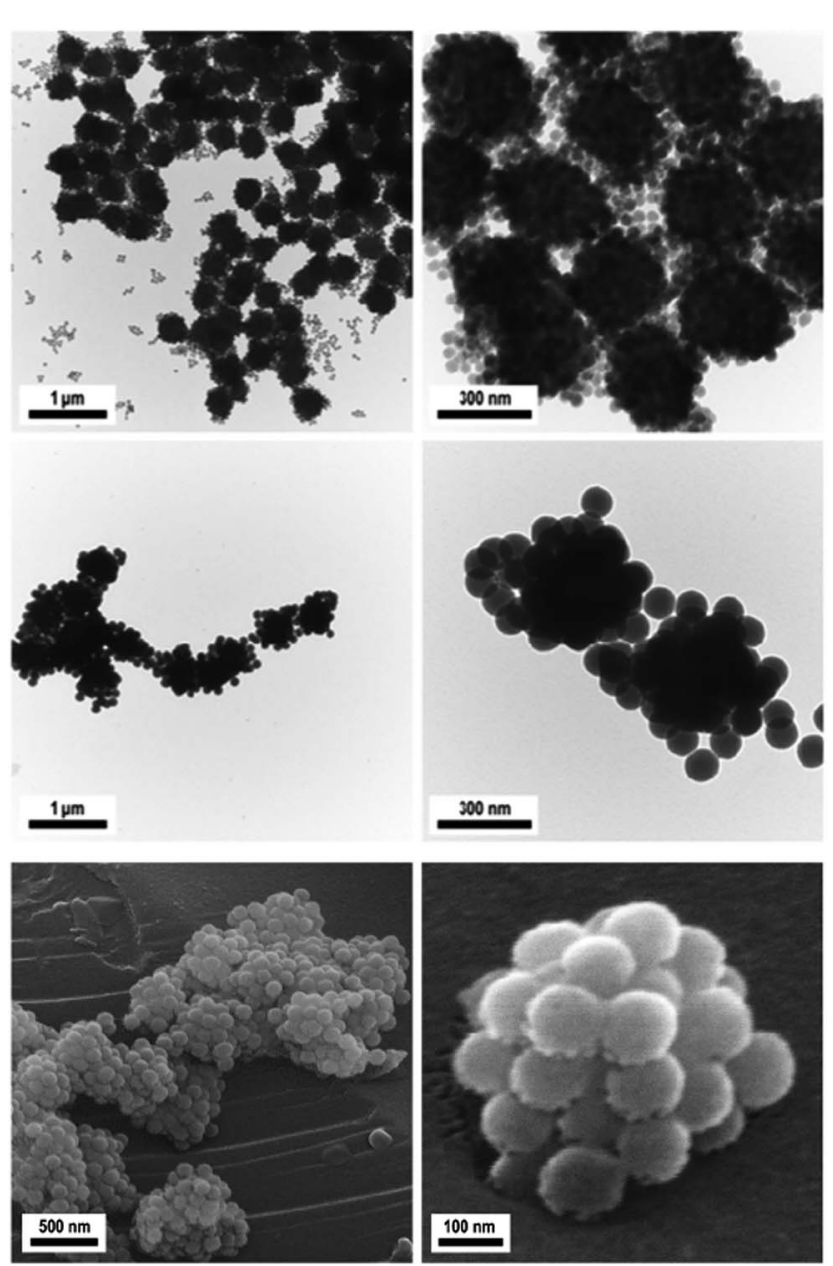

Fig. 5 TEM images of $348 \mathrm{~nm}$ PVPGE-containing organic core particles functionalized with $50 \mathrm{~nm}$ silica particles (top) and $120 \mathrm{~nm}$ silica particles (middle) and corresponding SEM images of PVPGE-containing organic core particles functionalized with $120 \mathrm{~nm}$ silica particles (bottom) 


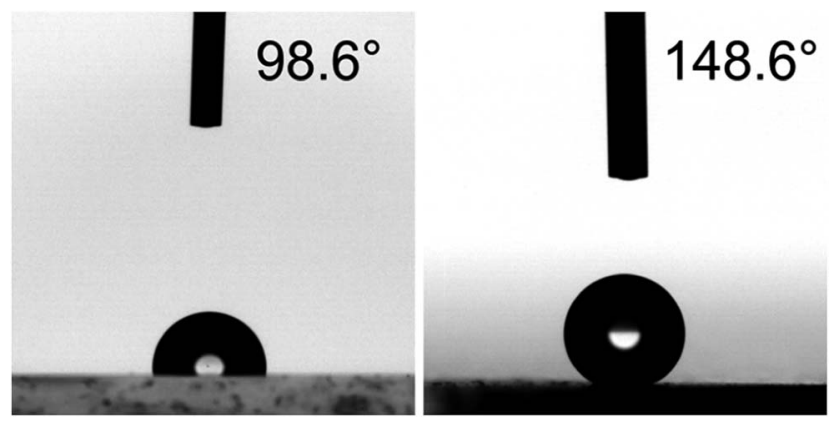

Fig. 6 Static contact angle measurements of water on hydrophobized glass wafer (left) and hydrophobized film obtained after deposition of PVPGE-containing hybrid raspberry particles (right), oxygen plasma treatment and silanization with $\mathrm{Me}_{2} \mathrm{SiCl}_{2}$.

the entire particle film as well as the particle structure remained stable, as proven by SEM measurements (Fig. S10†). In order to study the wetting properties of the silane-treated hybrid particle films, static CA measurements against water were carried out (Fig. 6).

Results of the static CA for the hydrophobized raspberry-type structures were directly compared to the hydrophobized glass wafers after functionalization with dichlorodimethylsilane and values were found to increase from $98.6^{\circ} \pm 3.1^{\circ}$ for the reference sample up to $148.6^{\circ} \pm 1.7^{\circ}$ for the raspberry-particle coated glass wafers. Noteworthy, CA measurements were carried out at 10 different positions of the glass wafer, revealing nearly identical CA values. These results evidence the suitability for herein prepared and investigated raspberry-type colloidal particles as highly water-repellent surface coatings.

In conclusion, a facile and straightforward synthetic procedure for the preparation of poly(vinylphenylglycidyl ether) (PVPGE)-containing core/shell latex particles has been developed that possess a high amount of reactive epoxy moieties at the surface. The presence of reactive epoxy functionalities was proven by (i) reaction with the Preussmann reagent (NBP) and determination of colored functional groups using UV/vis spectroscopy in anisole as an isorefractive solvent and (ii) by investigating the reaction of isophorone diamine (IPDA) with PVPGEcontaining core/shell particles via differential scanning calorimetry (DSC). The addition of silica nanoparticles with amine functionalities was employed for the preparation of raspberrylike organic-inorganic hybrid particles with organic core (348 nm) and smaller silica particles as shell. After surface deposition, plasma treatment and silanization, the raspberrylike particles deposited on glass substrates led to excellent static contact angles against water on the order of $150^{\circ}$, remarkably without the use of fluorinated compounds as hydrophobic coating agents. In summary, these findings represent a facile approach for the preparation of hierarchical particle architectures suitable for highly hydrophobic surfaces. Moreover, the synthesis of the novel raspberry-like architectures and coating strategies can be exploited as excellent and facile platform for the preparation of ultra-hydrophobic surfaces with adjustable surface roughness which is currently under investigation. Furthermore, this work evidences the potential of the novel bifunctional styrene monomer VPGE that can readily be combined with styrene and a broad range of other vinyl monomers. The highly epoxide-functional organic latex particles are also promising for photonic films, as toughening agents as well as materials for chemical sensing applications.

\section{Acknowledgements}

The authors thank Daniel Scheid for support for the particle film preparation, Jurema Schmidt (Juju) for help with particle synthesis and the group of Prof. Markus Biesalski from the Macromolecular Chemistry and Paper Department (TU Darmstadt) for help with CA measurements. We acknowledge the Landesoffensive zur Entwicklung WissenschaftlichÖkonomischer Exzellenz (LOEWE) of the State of Hesse through the research initiative Soft Control for ongoing financial support.

\section{Notes and references}

1 M. E. Davis, Nature, 2002, 417, 813-821.

2 C. Burda, X. Chen, R. Narayanan and M. A. El-Sayed, Chem. Rev., 2005, 105, 1025-1102.

3 Y. Zhao and L. Jiang, Adv. Mater., 2009, 21, 3621-3638.

4 C. G. Schäfer, S. Vowinkel, G. P. Hellmann, T. Herdt, C. Contiu, J. J. Schneider and M. Gallei, J. Mater. Chem. C, 2014, 2, 7960-7975.

5 W. Sun, S. Zhou, B. You and L. Wu, J. Mater. Chem. A, 2013, 1, 3146-3154.

6 F. Shi, Y. Song, J. Niu, X. Xia, Z. Wang and X. Zhang, Chem. Mater., 2006, 18, 1365-1368.

7 C. L. Feng, Y. J. Zhang, J. Jin, Y. L. Song, L. Y. Xie, G. R. Qu, L. Jiang and D. B. Zhu, Langmuir, 2001, 17, 4593-4597.

8 Y. Pei, J. Travas-Sejdic and D. E. Williams, Langmuir, 2012, 28, 8072-8083.

9 J. Hu, H. Meng, G. Li and S. I. Ibekwe, Smart Mater. Struct., 2012, 21, 053001.

10 J. Drelich, E. Chibowski, D. D. Meng and K. Terpilowski, Soft Matter, 2011, 7, 9804-9828.

11 R. N. Wenzel, Ind. Eng. Chem. Res., 1936, 28, 988-994.

12 A. B. D. Cassie and S. Baxter, Trans. Faraday Soc., 1944, 40, 0546-0550.

13 W. Ming, D. Wu, R. v. Benthem and G. d. With, Nano Lett., 2005, 5, 2298-2301.

14 W. Jiang, C. M. Grozea, Z. Shi and G. Liu, ACS Appl. Mater. Interfaces, 2014, 6, 2629-2638.

15 B. Peng, L. Tan, D. Chen, X. Meng and F. Tang, ACS Appl. Mater. Interfaces, 2012, 4, 96-101.

16 T. Ribeiro, C. Baleizão and J. Farinha, Materials, 2014, 7, 3881-3900.

17 L. A. Fielding, J. Tonnar and S. P. Armes, Langmuir, 2011, 27, 11129-11144.

18 H.-J. Tsai and Y.-L. Lee, Langmuir, 2007, 23, 12687-12692.

19 A. Schrade, Z. Cao, K. Landfester and U. Ziener, Langmuir, 2011, 27, 6689-6700.

20 Z. Nie, J. I. Park, W. Li, S. A. F. Bon and E. Kumacheva, J. Am. Chem. Soc., 2008, 130, 16508-16509. 
21 F. Tiarks, K. Landfester and M. Antonietti, Langmuir, 2001, 17, 5775-5780.

22 Y. Zhang, H. Chen, X. Shu, Q. Zou and M. Chen, Colloids Surf., A, 2009, 350, 26-32.

23 S.-W. Zhang, S.-X. Zhou, Y.-M. Weng and L.-M. Wu, Langmuir, 2005, 21, 2124-2128.

24 B. Zhao and M. M. Collinson, Chem. Mater., 2010, 22, 43124319.

25 H. Minami, Y. Mizuta and T. Suzuki, Langmuir, 2013, 29, 554-560.

26 M. Agrawal, A. Pich, S. Gupta, N. E. Zafeiropoulos, P. Formanek, D. Jehnichen and M. Stamm, Langmuir, 2010, 26, 526-532.

27 M. Agrawal, A. Pich, N. E. Zafeiropoulos, S. Gupta, J. Pionteck, F. Simon and M. Stamm, Chem. Mater., 2007, 19, 1845-1852.

28 C.-X. Zhao and A. P. J. Middelberg, RSC Adv., 2013, 3, 2122721230.

29 B. Chen, N. Pernodet, M. H. Rafailovich, A. Bakhtina and R. A. Gross, Langmuir, 2008, 24, 13457-13464.

30 H. Hlidkova, D. Horak, V. Proks, Z. Kucerova, M. Pekarek and J. Kucka, Macromol. Biosci., 2013, 13, 503-511.

31 P. Li, R. Xu, W. Wang, X. Li, Z. Xu, K. W. Yeung and P. K. Chu, Colloids Surf., B, 2013, 101, 251-255.

32 F. Limé and K. Irgum, Macromolecules, 2009, 42, 4436-4442.

33 Y. Lv, F. M. Alejandro, J. M. Frechet and F. Svec, J. Chromatogr. A, 2012, 1261, 121-128.

34 M. Ghaemy and S. Bekhradnia, J. Appl. Polym. Sci., 2010, 117, 467-472.

35 J. Lu, A. J. Easteal and N. Edmond, J. Appl. Polym. Sci., 2012, 126, 172-181.

36 S. Zhou, A. Bismarck and J. H. G. Steinke, J. Mater. Chem., 2012, 22, 18824-18829.

37 S. Sun, M. Zhang, H. Zhang and X. Zhang, J. Appl. Polym. Sci., 2011, 122, 2992-2999.

38 F. Radovanović, A. Nastasović, T. Tomković, D. VasiljevićRadović, A. Nešić, S. Veličković and A. Onjia, React. Funct. Polym., 2014, 77, 1-10.

39 Z. Luo, C. Zou, S. Syed, L. A. Syarbaini and G. Chen, Colloid Polym. Sci., 2011, 290, 141-150.
40 W. Zhao, R. J. Yang, T. T. Qian, X. Hua, W. B. Zhang and W. Katiyo, Int. J. Mol. Sci., 2013, 14, 12073-12089.

41 P. Tao, Y. Li, A. Rungta, A. Viswanath, J. Gao, B. C. Benicewicz, R. W. Siegel and L. S. Schadler, J. Mater. Chem., 2011, 21, 18623-18269.

42 P. Tao, A. Viswanath, Y. Li, R. W. Siegel, B. C. Benicewicz and L. S. Schadler, Polymer, 2013, 54, 1639-1646.

43 W. L. Zhang, S. H. Piao and H. J. Choi, J. Colloid Interface Sci., 2013, 402, 100-106.

44 J. Oh, J.-H. Lee, J. C. Koo, H. R. Choi, Y. Lee, T. Kim, N. D. Luong and J.-D. Nam, J. Mater. Chem., 2010, 20, 9200-9204.

45 X. Fan, X. Jia, H. Zhang, B. Zhang, C. Li and Q. Zhang, Langmuir, 2013, 29, 11730-11741.

46 N. Puretskiy and L. Ionov, Langmuir, 2011, 27, 30063011.

47 Y. Liu, M. Li and G. Chen, J. Mater. Chem. A, 2013, 1, 930-937. 48 K. Kunitsky, M. C. Shah, S. W. Shuey and M. E. Wagman, US 2008/0167433 A1 2008.

49 C. G. Schäfer, D. A. Smolin, G. P. Hellmann and M. Gallei, Langmuir, 2013, 29, 11275-11283.

50 D. Scheid, C. Lederle, S. Vowinkel, C. G. Schäfer, B. Stühn and M. Gallei, J. Mater. Chem. C, 2014, 2, 2583-2590.

51 C. G. Schäfer, M. Gallei, J. T. Zahn, J. Engelhardt, G. P. Hellmann and M. Rehahn, Chem. Mater., 2013, 25, 2309-2318.

52 M. Mazurowski, M. Gallei and M. Rehahn, ACS Macro Lett., 2012, 1, 1362-1366.

53 A. Hennig, H. Borcherding, C. Jaeger, S. Hatami, C. Würth, A. Hoffmann, K. Hoffmann, T. Thiele, U. Schedler and U. Resch-Genger, J. Am. Chem. Soc., 2012, 134, 8268.

54 M. Mazurowski, M. Gallei, J. Li, H. Didzoleit, B. Stühn and M. Rehahn, Macromolecules, 2012, 45, 8970-8981.

55 P. Schönherr, A. Seifert, R. Lungwitz, F. Simon, N. Moszner, P. Burtscher and S. Spange, Prog. Org. Coat., 2012, 75, 335343.

56 R. Preussmann, H. Schneider and F. Epple, Arzneim. Forsch., 1969, 19, 1059-1073.

57 E. Zyner, J. Graczyk and J. Ochocki, Pharmazie, 1999, 54, 945946. 